\title{
Atividade antimicrobiana de Aspidosperma nitidum benth (apocynaceae)
}

\author{
Antimicrobial activity of Aspidosperma nitidum benth (apocynaceae)
}

Actividad antimicrobiana de Aspidosperma nitidum benth (apocynaceae)

Heliton Patrick Cordovil Brígido ${ }^{1,2}$, Bárbara Tamires Chaves Ramos², Mipcia Katyucia Borges da $\mathrm{Paz}^{2}$, Mírian Letícia Carmo Bastos ${ }^{3}$, Maria Fâni Dolabela ${ }^{1 *}$.

\section{RESUMO}

Objetivo: Descrever a atividade antimicrobiana de Aspidosperma nitidum. Métodos: O extrato (EE) foi submetido à partição ácido-base obtendo-se as frações de alcaloides (FA) e de neutros (FN). Para verificar a atividade antimicrobiana, inicialmente realizou-se screening pelo teste de disco difusão em ágar frente às cepas de Sthaphylococcus aureus, Escherichia coli, Pseudomonas aeruginosa, Klebsiella pneumoniae e Proteus mirabilis. Para as amostras que apresentaram halo de inibição no teste de disco difusão em ágar, foi realizada a determinação da concentração inibitória mínima (CIM) pelo teste de microdiluição utilizando o MTT. Resultados: No teste de difusão em ágar, o EE, FA, FN inibiram o crescimento de bactérias $S$. aureus e E. coli. Não houve inibição do crescimento para as outras cepas. Na determinação da CIM, o EE, FA e FN apresentaram CIM de $1000 \mu \mathrm{g} / \mathrm{mL}$ para E. coli. Frente à cepa de S. aureus, o EE apresentou CIM de 250 $\mu \mathrm{g} / \mathrm{mL}$, sendo considerado moderadamente ativo, já as frações FA e FN foram considerados de fraca atividade, pois apresentaram CIM de $500 \mu \mathrm{g} / \mathrm{mL}$. Conclusão: A maior atividade antimicrobiana do extrato frente a $S$. aureus pode estar relacionada com alcaloides $\beta$-carbolínicos, ou com um possível sinergismo entre esta classe de alcaloides e alcaloides indólicos.

Palavras-chave: Aspidosperma, Alcaloides, Antibacteriano.

\begin{abstract}
Objective: Describe the antimicrobial activity of Aspidosperma nitidum. Methods: The ethanolic extract (EE) was subjected to acid-base fractionation aimed at obtaining alkaloid-rich fraction (FA) and neutral fraction (FN). For checking antimicrobial activity, a screening by diffusion disc test was performed against Sthaphylococcus aureus, Escherichia coli, Pseudomonas aeruginosa, Klebsiella pneumoniae and Proteus mirabilis. For samples that showed an inhibition halo in the agar diffusion disk test, a microdilution test in MTT was used to determine their minimum inhibitory concentration (MIC). RESULTS: In the agar diffusion test, EE, FA, and FN inhibited $S$. aureus and $E$. coli growth. There was no growth inhibition for the other strains. In MIC determination, the EE, FA and FN presented MIC of $1000 \mu \mathrm{g} / \mathrm{mL}$ against $E$. coli. In the $S$. aureus strain, the EE showed a MIC of $250 \mu \mathrm{g} / \mathrm{mL}$, considered moderately active, whereas the FA and FN fractions had low activity, as they presented a MIC of $500 \mu \mathrm{g} / \mathrm{mL}$. Conclusion: The Greater antimicrobial activity of the extract facing $S$. aureus may be available with $\beta$-carbolinic alkaloids, or with a possible synergism between this class of alkaloids and indole alkaloids.
\end{abstract}

Keywords: Aspidosperm, Alkaloids, Antibacterial.

\footnotetext{
1 Universidade Federal do Pará (UFPA), Belém - PA. *E-mail: fanidolabela20@gmail.com

${ }^{2}$ Faculdade Cosmopolita, Belém - PA.

${ }^{3}$ Núcleo de Pesquisa em Educação e Saúde na Amazônia (NUPESA), Tucuruí - PA.
} 


\section{RESUMEN}

Objetivo: Describir la actividad antimicrobiana de Aspidosperma nitidum. Métodos: El extracto etanólico (EE) se sometió a la partición ácido-base obteniendo las fracciones de alcaloides (FA) y neutrales (FN). Para verificar la actividad antimicrobiana, se realizó un cribado utilizando la prueba de disco de difusión de agar contra las cepas de Sthaphylococcus aureus, Escherichia coli, Pseudomonas aeruginosa, Klebsiella pneumoniae y Proteus mirabilis. Para las muestras que mostraron un halo de inhibición en la prueba de disco de difusión de agar, la determinación de la concentración inhibitoria mínima (MIC) se realizó mediante la prueba de microdilución usando MTT. Resultados: En la prueba de difusión en agar, EE, FA, FN inhibieron el crecimiento de las bacterias $S$. aureus y $E$. coli. No hubo inhibición del crecimiento para las otras cepas. Al determinar la MIC, EE, FA y FN presentaron una MIC de $1000 \mu \mathrm{g} / \mathrm{mL}$ para E. coli. La cepa frontal de S. aureus mostró una MIC EE $250 \mathrm{ug} / \mathrm{mL}$, y se considera moderadamente activa, ya que las fracciones FN y FA se consideraron actividad débil, mostraron una MIC de $500 \mu \mathrm{g} / \mathrm{mL}$. Conclusión: La mayor actividad antimicrobiana del extracto contra $S$. aureus puede estar relacionada con alcaloides $\beta$-carbolínicos, o con un posible sinergismo entre esta clase de alcaloides y alcaloides indólicos.

Palabras clave: Aspidosperm, Alcaloides, Antibacterianos.

\section{INTRODUÇÃO}

A antibioticoterapia é utilizada como primeira opção no tratamento de diversas infecções microbianas (BECKER K, et al., 2006). No entanto, o aumento da resistência aos antibióticos e outras drogas antimicrobianas é observado na atualidade (WHO, 2018). Segundo a Organização Mundial da Saúde (OMS), cerca de 700.000 pessoas morrem em decorrência da resistência bacteriana a esses medicamentos, configurando um grave problema de saúde pública, atingindo proporções mundiais, podendo ocasionar morte de 10 milhões de pessoas anualmente até o ano de 2050. Associado a isso, a maioria dos fármacos utilizados como antimicrobianos ocasionam efeitos nocivos ao organismo, comprometendo o bem-estar e a saúde (BERQUÓ LS, et al., 2004).

Nesse contexto, a busca por fármacos com ação antimicrobiana torna-se urgente, e as plantas medicinais podem fornecer alternativas promissoras para desenvolvimento dessas novas terapias (KLEIN T, et al., 2009). Dentre as plantas com atividade antimicrobiana, destacam-se espécies do gênero Aspidosperma (Apocynaceae) que apresentam derivados do loideschiquimato, especialmente taninos e alcaloides, metabólitos com grande potencial terapêutico (SIMINSKI T, et al., 2015). Ademais, espécies pertencentes a esta família caracterizam-se quimicamente pela ocorrência frequente de alcaloides (SCHRIPSEMA J, et al., 2004).

Espécies do gênero Aspidosperma são usadas pela medicina popular em doenças parasitárias como malária e leishmaniose. Também são usadas em diversas infecções e em feridas de difícil cicatrização (BOURDY G, et al., 2001; OLIVEIRA FQ, et al., 2003). No entanto, apesar do potencial terapêutico, espécies deste gênero carecem de estudos, como é o caso da Aspidosperma nitidum, popularmente conhecida como carapanaúba (Brasil) Jaroro hariraros, apokuita e padapan (Suriname), gabetillo (Bolívia), objeto de estudo do presente trabalho (FERREIRA-NETO WM, 1988).

A. nitidum é encontrada no continente americano, numa área que se estende do Panamá ao Brasil, sendo florescente principalmente entre os meses de julho a setembro. Morfologicamente esta espécie se caracteriza por árvores de 7 a 40 metros de altura, com tronco sulcado ou lamelado (FERREIRA-NETO WM, 1988). É uma planta bastante utilizada na medicina tradicional. Há relatos do uso em inflamações do útero e ovários, em problemas relacionados à diabetes e problemas do estômago. Também é usada contra câncer, e ainda como contraceptivo (RIBEIRO JELS, et al., 1999). Há também indicação para Malária e seu tratamento sintomático. Na Colômbia, o látex de $A$. nitidum é utilizado por índios para tratamento da hanseníase, contra febre e reumatismo (RIBEIRO JELS, et al., 1999; WENIGER B, et al., 2001).

Estudos fitoquímicos realizados em A. nitidum demonstraram o isolamento de vários alcaloides como: 10metoxi-18,19-diidrocorinanteol, corinanteol, aspidospermina, quebracamina, ioimbina, ácido harmanocarboxílico, ácido 3-metil-harmanocarboxílico, di-hidrocorinanteol, des-hidrositsirikina e braznitidumina (ARNDT RR et al., 1967; MARQUES MFS, et al., 1996; PEREIRA MM, et al., 2007). 
Apesar do vasto uso na medicina tradicional e da diversidade fitoquímica da espécie, nenhuma das abordagens apresentadas avaliou $A$. nitidum quanto ao seu potencial antimicrobiano, tornando essencial estudos que abordem essa questão. Sendo assim, o objetivo do trabalho consistiu em avaliar a atividade antimicrobiana do extrato etanólico e de suas frações obtidos a partir da casca de A. nitidum.

\section{MÉTODOS}

\section{Material vegetal}

Cascas do tronco de A. nitidum foram coletadas na rodovia estadual PA-150 ("coordenadas S $02^{\circ} 09^{\prime} 50.3^{\prime \prime}$ e W $\left.048^{\circ} 47^{\prime} 56.9^{\prime \prime}\right)$, no estado do Pará, em agosto de 2017. A exsicata encontra-se depositada em um Herbário do referido estado. O projeto está cadastrado na plataforma do sistema nacional de gestão e patrimônio genético e do conhecimento tradicional associado (SISGEN) sob inscrição A2C3188.

\section{Microrganismos}

Foram utilizados isolados clínicos de Sthaphylococcus aureus, Klebsiella pneumoniae, Pseudomonas aeruginosa, Proteus mirabilis e Escherichia coli gentilmente cedidas pelo Laboratório Beneficente de Belém (R. dos Mundurucus, 1818 - Batista Campos, Belém - PA, 66035-360), em ágar nutriente e mantidas em refrigerador a $8^{\circ} \mathrm{C}$ até a realização dos testes.

\section{Preparação do extrato e obtenção das frações}

As cascas de $A$. nitidum foram lavadas em água corrente e secas em estufa de ar circulado $\left(40^{\circ} \mathrm{C}\right.$, por 7 dias). $O$ material seco foi submetido à moagem em moinho de facas. $O$ pó da planta foi submetido à maceração com etanol a 96 GL (proporção 1:10). A solução etanólica foi filtrada e concentrada em evaporador rotatório sob pressão reduzida até total evaporação do álcool, obtendo-se o extrato etanólico seco (EE).

$\mathrm{O}$ EE $(5 \mathrm{~g})$ foi submetido à partição ácido: base, sendo solubilizado em etanol $(4,0 \mathrm{~mL})$ e adicionado solução aquosa de ácido clorídrico a $3 \%(7,5 \mathrm{~mL})$. Esta solução foi extraída com diclorometano (250 mL por 3 vezes), obtendo-se a fração de neutros (FN). A camada aquosa ácida foi alcalinizada com hidróxido de amônio a $10 \%\left(\mathrm{NH}_{4} \mathrm{OH}\right)$ até $\mathrm{pH}$ 9, seguida de nova extração com diclorometano ( $250 \mathrm{~mL}$ por 3 vezes), sendo obtida uma camada aquosa alcalina e uma camada orgânica (fração de alcaloides-FA).

\section{Atividade antimicrobiana}

\section{Avaliação Preliminar da atividade antimicrobiana}

Para avaliação da atividade antimicrobiana do extrato e suas frações (FA e FN), foi empregado o método de disco difusão em ágar. Esse ensaio serviu como screening para selecionar as amostras que obtiveram atividade antimicrobiana para posterior verificação da concentração inibitória mínima (CIM). No método de disco difusão, as culturas bacterianas desenvolvidas em caldo Mueller-Hinton por 24 horas foram diluídas (cerca de $10^{8} \mathrm{UFC} / \mathrm{mL}$ ) e semeadas por esgotamento total na superfície de ágar Mueller-Hinton. A seguir, discos de papel de filtro $(6 \mathrm{~mm})$ impregnados com as amostras (EE, FA e FN) a serem testados foram colocados sobre a superfície do ágar inoculado na dose de $1000 \mu \mathrm{g} /$ disco e incubados por 48 horas a $37^{\circ} \mathrm{C}$.

Como controle negativo foi utilizado discos impregnados com Dimetilsulfóxido (DMSO), solvente utilizado para solubilização das amostras. Como controles positivos foram utilizados os antibióticos Amoxicilina/Clavulanato e Gentamicina. Para leitura dos resultados, as amostras que apresentaram halo de inibição ao redor dos discos foram consideradas positivas e as que não apresentaram foram consideradas negativas (BAUER AW, et al., 1966; NCCLS, 2003).

\section{Determinação da concentração inibitória mínima (CIM)}

As amostras consideras positivas na atividade antimicrobiana de avaliação preliminar foram submetidas à determinação da concentração inibitória mínima (CIM) pela técnica de microdiluição em caldo. As culturas bacterianas desenvolvidas em caldo Muller-Hinton e diluídas (cerca de $10^{8} \mathrm{UFC} / \mathrm{mL}$ ) foram colocadas em placas de 96 poços previamente dosificadas com as amostras (EE, FA e FN) nas concentrações de 1000 
$\mu \mathrm{g} / \mathrm{mL}, 500 \mu \mathrm{g} / \mathrm{mL}, 250 \mu \mathrm{g} / \mathrm{mL}, 125 \mu \mathrm{g} / \mathrm{mL}, 62,5 \mu \mathrm{g} / \mathrm{mL}, 31,5 \mu \mathrm{g} / \mathrm{mL}, 15,625 \mu \mathrm{g} / \mathrm{mL}$. Como controle negativo utilizou-se o DMSO (CLSI, 2015). Como controle positivo amoxicilina/clavulanato para $S$. aureus e gentamicina para E.coli nas concentrações de $250 \mu \mathrm{g} / \mathrm{mL}, 125 \mu \mathrm{g} / \mathrm{mL}, 62,5 \mu \mathrm{g} / \mathrm{mL}, 31,5 \mu \mathrm{g} / \mathrm{mL}, 15,625 \mu \mathrm{g} / \mathrm{mL}$, $7,814 \mu \mathrm{g} / \mathrm{mL}, 3,906 \mu \mathrm{g} / \mathrm{mL}$. As placas foram incubadas a $37^{\circ} \mathrm{C}$ por 24 horas.

Após período de incubação, foram acrescentados $10 \mu \mathrm{L}$ de solução de MTT $(5 \mathrm{mg} / \mathrm{mL})$, e as placas foram novamente incubadas por 4h. A CIM é a menor concentração das amostras testadas onde não houve crescimento bacteriano visível (CHAUD MV, et al., 2005). Os resultados da CIM foram interpretados como: $\mathrm{CIM}<100 \mu \mathrm{g} / \mathrm{mL}$ : ativo, entre 100 a $500 \mu \mathrm{g} / \mathrm{mL}$ : moderadamente ativo, entre 500 a $1000 \mu \mathrm{g} / \mathrm{m}$ : fraca atividade e acima de $1000 \mu \mathrm{g} / \mathrm{mL}$ : inativo (DALL' AGNOL R, et al., 2003; TANAKA JCA, et al., 2005).

\section{RESULTADOS}

No processo de extração do pó das cascas de $A$. nitidum através da maceração com etanol, obteve-se um rendimento de $8,78 \%$ de extrato $(87,8 \mathrm{~g})$. $O$ fracionamento desse extrato por partição ácido-base, gerou as frações de neutros $(24,73 \%)$ e alcaloides $(20,56 \%)$.

$\mathrm{Na}$ avaliação preliminar da atividade antimicrobiana do extrato e suas frações (FA e FN) no teste de disco difusão em ágar sobre os microrganismos, verificou-se que todas amostras foram capazes de inibir 0 crescimento de $S$. aureus e E. coli, pois observou-se halo de inibição ao redor dos discos, logo foram consideras positivas (Tabela 1). Por outro lado, frente às cepas de $P$. aeruginosa, $P$. mirabilis e $K$. pneumoniae, tanto o extrato quanto suas frações (FA e FN) foram considerados negativos, pois não houve formação de halo de inibição ao redor dos discos (Tabela 1).

Tabela 1 - Atividade antimicrobiana das amostras obtidas de Aspidosperma nitidum.

\begin{tabular}{ccccc}
\hline Microrganismos & \multicolumn{4}{c}{ Amostras $(\mathbf{1 0 0 0} \boldsymbol{\mu g} / \mathbf{d i s c o})$} \\
\cline { 2 - 5 } & EE & FA & FN & CP* $^{*}$ \\
Escherichia coli & + & + & + & + \\
Kthaphylococcus aureus & + & + & + & + \\
Psebsiella pneumoniae & - & - & - & + \\
Proteus mirabilis & - & - & - & + \\
\hline
\end{tabular}

Legenda: EE: extrato etanólico; FA: fração de alcaloides; FN: fração de neutros; CP: controle positivo *Amoxicilina/CLAV e Gentamicina; +: Positivo; -: Negativo.

Fonte: Brígido HPC, et al., 2020.

Na determinação da concentração inibitória mínima, o EE obtido da casca de A. nitidum apresentou CIM de $250 \mu \mathrm{g} / \mathrm{mL}$ frente à cepa de $S$. aureus, sendo considerado moderadamente ativo. Já as frações FA e FN apresentaram CIM de $500 \mu \mathrm{g} / \mathrm{mL}$, possuindo fraca atividade antimicrobiana. Em E. coli, tanto o EE quanto as frações FA e FN apresentaram fraca atividade, pois apresentaram CIM de $1000 \mu \mathrm{g} / \mathrm{mL}$ (Tabela 2).

Tabela 2: Concentração Inibitória Mínima (CIM) das amostras obtidas de Aspidosperma nitidum.

\begin{tabular}{ccccc}
\hline \multirow{2}{*}{ Microrganismos } & \multicolumn{4}{c}{ Amostras $(\boldsymbol{\mu g} / \mathbf{m L})$} \\
\cline { 2 - 5 } & EE & FA & FN & CP $^{*}$ \\
Escherichia coli & 1000 & 1000 & 1000 & 3,906 \\
Sthaphylococcus aureus & 250 & 500 & 500 & 3,906
\end{tabular}

Legenda: EE: extrato etanólico; FA: fração de alcaloides; FN: fração de neutros; CP: controle positivo *Amoxicilina/CLAV e Gentamicina.

Fonte: Brígido HPC, et al., 2020.

\section{DISCUSSÃO}

A investigação da atividade antimicrobiana do extrato etanólico e de suas frações obtidos a partir da casca de $A$. nitidum, demonstrou que a planta possui atividade antimicrobiana promissora contra cepas de $S$. aureus e E. coli. Tratando-se da questão relacionada à resistência dos microrganismos aos medicamentos disponíveis, esse resultado serve como base para futuros estudos para o desenvolvimento de fármacos antimicrobianos. 
Uma característica importante ao se analisar antimicrobianos é que o perfil de inibição de crescimento bacteriano pode variar entre diferentes microorganismos quando em contato com diferentes substâncias usadas como antimicrobianas (VOLKART PA, et al., 2017). Neste estudo, inicialmente, foi avaliado o comportamento de 5 cepas ( $S$. aureus, E. coli, P. aeruginosa, $P$. mirabilis e K. pneumoniae) com relação ao crescimento e formação de halos de inibição ao redor dos discos contendo amostras de A. nitidum (EE, FN e FA) através do teste de disco difusão em ágar (Tabela 1).

Nesse ensaio, apenas $S$. aureus e E. coli foram sensíveis a todas as amostras testadas (EE, FA e FN), inibindo o crescimento de $S$. aureus e $E$. coli no teste de disco difusão em ágar (Tabela 1). Atividade semelhante foi observada por outros autores, utilizando outras espécies do gênero Aspidosperma (AGRIPINO DG, et al., 2004; TANAKA JCA, et al., 2006; OLIVEIRA VB, et al., 2009).

Tal atividade foi relacionada aos alcaloides, por alguns autores. Tanaka JCA, et al. (2006), por exemplo, sugerem que a atividade do extrato metanólico da casca de Aspidosperma ramiflorum frente à cepa de $S$. aureus esteja relacionada com a alta quantidade de alcaloides, dentre eles o alcaloide isolado da espécie, a ramiflorina, que também apresentou atividade frente a esta cepa. Estudo realizado por Oliveira VB, et al. (2009) atribuiu a atividade antimicrobiana de Aspidosperma excelsum frente à cepa de E. coli aos alcaloides indólicos.

Quando analisamos as outras cepas Gram-negativas ( $P$. aeruginosa, P. mirabilis e K. pneumoniae), observamos que o extrato e suas frações foram considerados inativos (Tabela 1). Outros estudos também demonstram a inatividade das Aspidospermas frente a bactérias gram-negativas (OLIVEIRA VB, et al., 2009; PESSINI GL, 2012). O extrato etanólico e frações obtidas de A. tometosun, A. macrocarpum e $A$. pyrifolium foram inativas em $P$. aeruginosa, entretanto, também foram inativas em $E$. Coli (PESSINI GL, 2012). Outro estudo também mostra que o extrato bruto obtido de A. ramiflorum, A. pyricolum, A. olivaceum, A. dispermum, A. pyrifolium, A. polyneuron foram inativos contra P. Aeruginosa (OLIVEIRA VB, et al., 2009).

A inatividade da ação antimicrobiana de cepas Gram-negativas no teste de disco-difusão pode estar relacionada com diferenças estruturais entre os tipos de bactérias Gram-positivas e Gram-negativas, que conferem diferentes graus de permeabilidade de substâncias na parede bacteriana, sendo que, bactérias Gram-negativas possuem maior seletividade quanto à passagem de substâncias. A presença de estruturas como membrana externa e cápsula, presença de porinas ou, até mesmo, não transcrição de canais de porinas - a partir de mecanismos de resistência bacteriana - que podem dificultar a ação de substâncias ativas das amostras investigadas (KONEMAN EW, et al., 1999; BRUNTON L, HILAL-DANDAN R, 2015).

Além do perfil antimicrobiano ser variável entre microrganismos, como comentado anteriormente, neste estudo, também percebe-se diferença de perfil de atividade antimicrobiana relacionada às amostras estudadas, as quais variaram na determinação da concentração inibitória mínima, variando de atividade moderada (EE - CIM de $250 \mu \mathrm{g} / \mathrm{mL}$ para $S$. aureus) à atividade fraca (FA e FN - CIM de $500 \mu \mathrm{g} / \mathrm{mL}$ para $S$. aureus; EE; FA e FN - atividade antimicrobiana fraca para E. coli). Primeiramente, observa-se que, além do tipo de metabolito secundário presente (alcaloides), também, pode-se observar a interferência da concentração de metabólitos na amostra.

Portanto, outra hipótese para a inatividade da ação antimicrobiana em tais cepas (Tabela 2) pode estar relacionada com a concentração de substâncias ativas presente nas amostras, ou seja, o extrato e frações FA e FN podem apresentar baixa concentração de compostos ativos ou ainda, não apresentar nenhuma molécula ativa sobre as cepas. Essa característica pode estar relacionada com o fracionamento das amostras obtidas, uma vez que o fracionamento pode aumentar a atividade de compostos químicos ativos (GALUCIO NCR, 2014) e poderá também diminuir tal atividade.

O presente estudo mostrou que o fracionamento do extrato etanólico não contribuiu para a atividade antimicrobiana na cepa em questão, pois, ao analisarmos o seu fracionamento, tanto a fração rica em alcaloides (FA) quanto a $\mathrm{FN}$ apresentaram CIM de $500 \mu \mathrm{g} / \mathrm{mL}$ (Tabela 2). Ao analisarmos $E$. coli frente às amostras de A. nitidum, tanto o EE quanto suas frações FA e FN apresentaram atividade fraca (CIM: 1000 $\mu \mathrm{g} / \mathrm{mL}$; Tabela 2). A E. coli também faz parte das bactérias Gram-negativas e esse achado pode estar relacionado a estrutura anatômica e fisiológica desse tipo de bactéria, como citado anteriormente. 
Diante do exposto, observa-se ação antimicrobiana promissora para o EE - o qual apresentou atividade antimicrobiana no teste de difusão em ágar e foi ensaiado para determinação da CIM para cada uma das espécies bacterianas (OSTROSKY EA, et al., 2008) e mostrou melhor CIM frente a cepa de $S$. aureus (CIM: $250 \mu \mathrm{g} / \mathrm{mL}$; Tabela 2).

Assim como nesse estudo, a família Apocynaceae mostra ação antimicrobiana quando estudada por outros autores. Relacionado ao gênero Aspidosperma, muitos autores relatam tal atividade tanto para bactérias Gram positivas quanto para Gram negativas. Conegero LS, et al. (2003), ao avaliar a atividade do extrato bruto metanólico de $A$. glandulosa na avaliação da CIM, verificou-se moderada atividade (125 a 250 $\mu \mathrm{g} / \mathrm{mL})$.

O extrato bruto etanólico da casca da raiz de $A$. tomentosum demonstrou atividade antibacteriana fraca sobre $S$. aureos e $B$. subtilis e a fração de alcaloides do caule de $A$. pyrifolium apresentou atividade moderada para $S$. áureos e B. subtilis. (PESSINI GL, 2012). O extrato metanólico das cascas do caule de $A$. ramiflorum demonstrou atividade moderada em $B$. subtilis e $S$. áureos e E. faecalis (TANAKA JCA, et al, 2006). O extrato bruto etanólico de $A$. pyricolum e $A$. Olivaceum foram moderadamente ativos contra cepas de $B$. subitilis (OLIVEIRA AJB, 2009).

Relacionado a outros gêneros de Apocynaceae, essa ação também é relatada. Brandão DLN, (2012) verificou que o extrato e fração de alcaloides obtidos de Geissospermum vellosii apresentaram CIM de 125 $\mu \mathrm{g} / \mathrm{mL}$ frente a S. Aureus. Gonçalves DM, et al. (2011) demonstraram que o extrato de obtido de Tabernaemontana catharinensis teve atividade antimicrobiana in vitro frente a $S$. aureus e $P$. aeruginosa.

Dando enfoque aos metabólitos presentes em espécies de Apocynaceae, verifica-se constantemente a presença de alcaloides como constituintes ativos. Tabernaemontana catharinensis, por exemplo, possui composição química rica em alcalóides indólicos, mostrando o potencial biológico desses constituintes. Os alcaloides indólicos extraídos de Aspidosperma marcgravianum exibiram atividade antimicrobiana e citotóxica. Assim como, alcaloides indólicos isolados de Aspidosperma excelsum Benth apresentaram atividade antimicrobiana frente a Bacillus subtilis e Staphylococcus aureus (PEREIRA MM, et al., 2007).

Além disso, há estudos que evidenciam os alcaloides como compostos ativos para atividade antimicrobiana. $\mathrm{Na}$ avaliação da atividade antimicrobiana, da espécie Zanthoxylum stelligerum, por exemplo, houve inibição do crescimento in vitro de microrganismos e os autores indicaram a substância pura diidroqueleritrina, um alcalóide benzofenantridínico, como uma das substâncias ativas responsáveis por esta atividade. Essa espécie apresentou uma discreta atividade frente a cepa de $S$. aureus. $O$ alcaloide diidroqueleritrina exibiu um espectro de ação mais amplo que o perfil do extrato que o originou, o extrato metanólico da raiz da planta. Também houve atividade antimicrobiana frente a cepas Gram positivas e E. coli, uma bactéria Gram negativa (SILVA CV, et. al, 2010).

No presente estudo, a partir dos resultados positivos para CIM, podemos considerar que a ação apresentada pode estar relacionada com os metabólitos presentes em $A$. nitidum. Estudos fitoquímicos demonstraram que $A$. nitidum é constituída majoritariamente por alcaloides indólicos e alcaloides $\beta$ carbolínicos (ARNDT RR et al., 1967; MARQUES MFS, et al., 1996; PEREIRA MM, et al., 2007).

Brígido HPC, (2019) analisando a composição do EE, FA e FN obtido da casca de A. nitidum em cromatografia líquida de alta eficiência (CLAE-DAD), verificou que extrato etanólico apresentou compostos sugestivos de alcaloides $\beta$-carbolínicos e de alcaloides indólicos, a fração de alcaloides e a fração de neutros apresentaram compostos sugestivos apenas de alcaloides indólicos, mostrando que o fracionamento do extrato por partição ácido: base não foi eficiente para separação de alcaloides $\beta$-carbolínicos para estas frações. Neste contexto, tais resultados levantam a hipótese de que a maior atividade antimicrobiana do extrato frente à cepa de $S$. aureus pode estar relacionada com alcaloides $\beta$-carbolínicos, ou com um possível sinergismo entre esta classe de alcaloides e alcaloides indólicos.

Apesar de os resultados não se mostrarem inicialmente atrativos para a atividade antimicrobiana nesse estudo, mostram-se promissores, já que espécies de Aspidosperma são usadas na medicina tradicional para o tratamento de feridas, o que instiga a investigação sobre tal uso, sobretudo, por apresentarem metabólitos 
com diversas atividades biológicas, como é o caso dos alcaloides. Além disso, a investigação bacteriana dessas espécies de Aspidosperma podem ser averiguadas novamente, com fracionamento mais detalhado e realizado frente a outras cepas de bactérias. Devendo-se considerar também estudos que evidenciem fatores relacionados ao organismo, mecanismo de ação, até mesmo para suprir mecanismos de resistência bacteriana ou possível uso como adjuvante nos tratamentos e sinergismo farmacológico.

Tais resultados levantam a importância da pesquisa realizada a partir de produtos naturais. Sendo essencial que estudos preliminares de ação antimicrobiana sejam realizados para levar ao direcionamento estudos minuciosos de investigação fitoquímica. Relacionado ao estudo da espécie em questão, é primordial saber se há a presença de alcaloides já isolados em estudos anteriores em outras espécies de Apocynaceae e, futuramente, contribuir para a pesquisa de novos fármacos com ação antimicrobiana, sendo o primeiro relato de estudo de ação antimicrobiana de $A$. nitidum.

\section{CONCLUSÃO}

Foi demonstrado que o extrato e suas frações (FA e FN) obtidos de A. nitidum possuem potencial antimicrobiano contra bactérias Gram-negativas e Gram-positivas. Tal atividade pode estar relacionada com a presença de alcaloides. Sendo assim, os resultados obtidos estimulam a continuidade do estudo, visando o isolamento de substâncias, na perspectiva de revelar um composto com alta atividade antimicrobiana.

\section{AGRADECIMENTOS E FINANCIAMENTO}

Os autores agradecem a Faculdade Cosmopolita, ao Laboratório Beneficente de Belém e ao Laboratório de Farmacologia e Doenças Negligenciadas (LFDN) da Universidade Federal do Pará.

\section{REFERÊNCIAS}

1. AGRIPINO DG, et al. Screening of Brazilian plants for antimicrobial and NA-damaging activities. I. Atlantic rain forestEcological station Juréia-Itatins. Biota Neotropica, 2004; 4(2): 1-15.

2. ARNDT RR, et al. Alkaloid studies- LVIII: the alkaloids of six Aspidosperma species. Phytochemistry, 1967; 6(12): 1653-58.

3. BAUER AW, et al. Antibiotic susceptibility testing by a standardized single disk method. American Journal of Clinical Pathology, 1966; 45(4): 493-6.

4. BECKER K, et al. Infectious diseases - a global challenge. International Journal of Medical Microbiology, 2006; 296: 179-185.

5. BERQUÓ LS, et al. Utilização de medicamentos para tratamento de infecções. Revista de Saúde Pública, 2004; 38(3): 358-64-528.

6. BOURDY G, et al. A search for natural bioactive compounds in Bolivia through a multidisciplinary approach. Part VI. Evaluation of the antimalarial activity of plants used by the tacana indians. Journal of Ethnopharmacology, 2001; 77(1): 91-8.

7. BRANDÃO DLN. Portulaca pilosa L. e Geissospermum vellosii. Estudos Botânicos, Farmacognósticos, Fitoquímicos e Atividades Biológicas. Dissertação (Mestrado em Ciências Farmacêuticas) - Instituto de Ciências da Saúde. Universidade Federal do Pará, Belém, 2012.

8. BRÍGIDO HPC. Atividade Antileishmania in vivo da Casca de Aspidosperma nitidum Benth. Ex Müll. Arg. (Apocynaceae). Tese (Doutorado em Inovação Farmacêutica). - Instituto de Ciências da Saúde. Universidade Federal do Pará, Belém, 2019; 70 p.

9. BRUNTON LL, HILAL-DANDAN R. Princípios gerais do tratamento antimicrobiano. In: manual de farmacologia e terapêutica de GOODMAN \& GILMAN. 2 ed. Porto Alegre (RS): Mc Graw Hill/Artmed, 2015; 829-833.

10. CHAUD MV, et al. Avaliação da atividade antimicrobiana de extratos vegetais. Revista Brasileira de Farmacognosia, 2005; 15(4): 316-320.

11. CLSI, Clinical and Laboratory Standards Institute. Methods for dilution antimicrobial susceptibility test for bactéria that grow aerobically. Wayne, CLSI, 2015; 10th ed. M07-A10.

12. CONEGERO LS, et al. Constituintes químicos de Alchornea glandulosa (Euphorbiaceae). Química nova, 2003; 26(6): 825-7.

13. DALL' AGNOL R, et al. Antimicrobial activity of some Hypericum species. Phytomedicine, 2003; 10(6-7): 511-6.

14. FERREIRA-NETO WM. Aspidosperma Mart., norm. cons. (Apocynaceae): estudos taxonômicos. Tese de Doutorado. Instituto de Biologia, Universidade Estadual de Campinas, Campinas, 1988; 431p.

15. GALUCIO NCR. Estudos fitoquímicos, Citotoxicidade e Genotoxicidade de Eleutherine Plicata Herb., Dissertação (Mestrado em Ciências Farmacêuticas) - Instituto de Ciências da Saúde. Universidade Federal do Pará, Belém, 2014; $90 \mathrm{p}$. 
16. GONÇALVES DM, et al. Avaliação da atividade antimicrobiana in vitro do extrato de Tabernaemontana catharinensis A. DC Revista Brasileira Plantas Medicinais, 2011; 13 (2): 197-202.

17. KLEIN T, et al. Fitoterápicos: Um mercado promissor. Revista de Ciências Farmacêuticas Básica e Aplicada, 2009; $30(3): 241-8$.

18. KONEMAN EW, et al. Diagnóstico microbiológico texto e Atlas Color. 5. Ed. Buenos Ayres: Panamerica, 1999.

19. MARQUES MFS, et al. Indole alkaloids from Aspidosperma ramiflorum. Phytochemistry, 1996; 41(3): 963-7.

20. NCCLS. Performance Standards for Antimicrobial Disk Susceptibility Tests; Approved Standard. NCCLS document M2-A8 (ISBN 1-56238-485-6). 2003; Pennsylvania. 8th ed. 19087-1898.

21. OLIVEIRA FQ, et al. Potencial das plantas medicinais como fonte de novos antimaláricos: espécies indicadas na bibliografia etnomédica brasileira. Revista Brasileira de Plantas Medicinais, 2003; 5(2): 23-31.

22. OLIVEIRA VB, et al. Atividade biológica e alcaloides indólicos do gênero Aspidosperma (Apocynaceae): uma revisão. Revista Brasileira de Plantas Medicinais, 2009; 11(1): 92-9.

23. OLIVEIRA AJB, et al. Preliminary studies on the antibacterial activity of ethanol crude extracts and alkaloids from species of Aspidosperma. Pharmaceutical Biology; 2009; 47(1): 1085-1089.

24. STROSKY EA, et al. Métodos para avaliação da atividade antimicrobiana e determinação da Concentração Mínima Inibitória (CMI) de plantas medicinais. Revista Brasileira de Farmacognosia, 2008; 18(2): 301-307.

25. PEREIRA MM, et al. Alcaloides indólicos isolados de espécies do gênero Aspidosperma (Apocynaceae). Revista Química Nova, 2007; 30(4)4: 970-983.

26. PESSINI GL. Evaluation of antimicrobial activity of three Aspidosperma species. Pharmacology online 2012; 1(1): 112119.

27. RIBEIRO JELS, et al. Guia de identificação das plantas vasculares de uma floresta de terra-firme na Amazônia Central. 19. ed. Manaus: Midas Printing, 1999; 568-81p.

28. SCHRIPSEMA J, et al. Alcaloides indólicos in Aspidosperma. In: SIMÕES CMO, et al. Farmacognosia: da planta ao medicamento. 2004. Porto Alegre/ Florianópolis: 689-716.

29. SILVA CV, et al. Avaliação da atividade antimicrobiana de duas espécies de Rutaceae do Nordeste brasileiro. Brazilian Journal of Pharmacognosy, 2010; 20(3): 355-360.

30. SIMINSKI T, et al. Avaliação da atividade Antimicrobiana das espécies Aspidosperma nitidum, Annona crassiflora e Annona mucosa frente cepas ATCC. Enciclopedia Biosfera, 2015.

31. TANAKA JCA, et al. Constituintes químicos de Luehea divaricata Mart. (Tiliaceae). Química Nova, 2005; 28(5).

32. TANAKA JCA, et al. Antibacterial activity of índole alkaloids from Aspidosperma ramiflorum. Brazilian Journal of Medical and Biological Research, 2006; 39(3): 387-391.

33. VOLKART PA, et al. Avaliação da susceptibilidade e resistência bacteriana aos agentes controladores do crescimento de uso hospitalar e industrial. Arquivos de Ciências da Saúde da UNIPAR, 2017; 21(1): 25-32.

34. WENIGER B, et al. Antiprotozoal activities of Colombian plants. Journal of Ethnopharmacology, 2001 78(2-3):193-200

35. WHO. Antimicrobial resistance, 2018. Disponível em: https://www.who.int/news-room/fact-sheets/detail/antimicrobialresistance. 